\title{
Study of Analytical Methods for Determining 16 Organophosphorus Pesticide Residues in Panax Notoginseng and Aucklandia Lappa Decne by Automated Solid-Phase Extraction System and GC/MS
}

\author{
$\mathrm{Na} \mathrm{Wu}$ \\ Honghe University \\ Gaozhang Gou \\ Honghe University \\ Dachao Liang \\ Honghe University \\ Jinru Ruan \\ Hong He Institute for Food and Drug Control \\ Wei Liu \\ Honghe University \\ Shaoping Feng ( $\nabla$ shaopingfeng@126.com ) \\ Honghe University
}

\section{Research Article}

Keywords: Comparative study, Sample preparation methods, Organophosphorus pesticide residues, Panax notoginseng and Aucklandia lappa decne, Automated solid-phase extraction system, Gas chromatography-mass spectrometry

Posted Date: February 1st, 2021

DOI: https://doi.org/10.21203/rs.3.rs-150299/v1

License: (c) (1) This work is licensed under a Creative Commons Attribution 4.0 International License. Read Full License 


\section{Abstract}

A fast and sensitive multi-residue analytical method was developed for the determination of organophosphorus pesticide residues in Panax notoginseng and Aucklandia lappa decne by comparing an ultrasonic extraction and homogenate extraction approach coupled with automated solid-phase extraction system and gas chromatography-mass spectrometry (GC/MS). Various factors affecting the homogenate extraction and purification efficiency were investigated in detail. The optimum conditions for homogenate extraction of organophosphorus pesticide residues from Panax notoginseng and Aucklandia lappa decne were $60 \mathrm{~mL}$ dichloromethane, $10000 \mathrm{r} / \mathrm{min}$ of extracting revolution speed and 2 min of extracting time. The extracts were cleaned up by automated solid-phase extraction system and determined by GC/MS. Under the optimum conditions, the results of the homogenate extraction approach were compared with those of the ultrasonic extraction, and the homogenate extraction approach was used to extract the samples. The average recoveries of the method by a homogenate extraction approach coupled with automated solid-phase extraction system and GC/MS ranged from $72.56 \%$ to $96.70 \%$, and RSDs were $0.05 \sim 0.14 \%$, which allowed the determination of 16 organophosphorus pesticide residues in Panax notoginseng and Aucklandia lappa decne. The results indicated that the proposed method can apply for the determination of organophosphate pesticides residues in Panax notoginseng and Aucklandia lappa decne with high accuracy and precision.

\section{Introduction}

Pesticide residues refer to pesticides, their toxic metabolites and degradation products and any impurities that may live on or in an biosome, farm products, or the environment after pesticides are used ${ }^{1-3}$.

Sometimes pesticide residues are inevitable; however, once the residue reaches its limitation, it can make a series of danger to humans and animals or other organism in the ecological system by the food cycle ${ }^{4}$. The organophosphate pesticides (OPPs) were widely used and leaded to the repeated incidents of poisoning, and therefore, self-poisoning with organophosphate pesticides is a significant global health problem ${ }^{5-7}$. Hence, a ban was imposed on the use of toxic pesticides, including OPPs, such as methamidophos, parathion, monocrotophos, methyl parathion, etc ${ }^{8-10}$.

The rules linking pesticides on plants and in agricultural products have be set up so far by a lot of international organizations and countries. With the remarkable enhancements in testing methods and analytical techniques of pesticide residues and the extensive attention of consumer safety in recent years, the developed countries have become strictly monitoring both the terms of pesticide class or amount and maximum residue levels (MRLs) in foodpesticide residues ${ }^{11-14}$. Multiresidue analytical systems, which have been developed worldwide, can simultaneously determine over three hundred pesticides ${ }^{15}$. The MRLs of 9 organochlorines (OCPs) were prescriptive in the Chinese pharmacopoeia, which involved benzene hexachloride (BHC), dichlorodiphenyltrichloroethane (DDT) and pentachloronitrobenzene (PCNB). Radix et Rhizoma Glycyrrhizae and Radix Astragali in the Pharmacopoeia of the People's Republic of China (Ch.P) were involved ${ }^{16}$. The first draft of the quality standards from china in July 2001, which had something to do with green professional standards for import and export of officinal plants and products, was issued and enforced in the ministry of foreign trade and economic cooperation. The 9 OCPs and aldrin were included according to the standards. The analytical methods of 3 pyrethroids and 12 OPPs were supplied separately in the Ch.P, but MRLs were not prescriptive. In order to improve the international regulations and standards of pesticide residues from Chinese traditional medicine, further research have been needed ${ }^{17}$. The main food safety issues focused on pesticide residues. Especially the MRLs of pesticide residues from many countries and health organizations had published. People have paid a more attention to analytical methods of remnant pesticides, so the analytical methods possessing sureness, simpleness, speediness, relatively steady, highly sensitive and repeatability is required. The sample pre-treatment and detected techniques have a crucial effect on the accuracy and precision of the analytical methods.

The sample pre-treatment techniques include liquid-liquid extraction, liquid-solid extraction, shaking extraction, soxhlet extraction, supercritical fluid extraction, pressurized solvent extraction, microwave-assisted extraction, ultrasound-assisted extraction, gel permeation chromatography, solid phase extraction, solid phase microextraction, molecularly imprinted polymers, matrix solid-phase dispersion, QuEChERS, cloud-point extraction, liquid phase microextraction, etc ${ }^{18-22}$. Automated solid-phase extraction system achieves artificial intelligence in the sample pre-treatment techniques.

With the significantly improving requirements in environmental quality and food safety, quantity of submitted test samples is increasing. Hence, this increase requires that the analytical method is fast, ingenious, accurate, and convenient. In order to accelerate the process of internationalizing and modernizing traditional Chinese herbal medicine, analysis methods, including GC,LC,GC/MS,LC/MS,SFC,CE,CE/MS and ELISAs for the detection of pesticide residues have been used to determine pesticide residues ${ }^{23-26}$. Great progress has been made on new analytical methods and techniques at present, especially in pesticides multiresidue analyses method. The analysises of multipesticide residues from the same class of pesticid and a trial sample of new single pesticides in Panax notoginseng were also reported ${ }^{27,28}$.

Panax notoginseng belonging to araliaceae species which mainly produced in the any counties of WenShan prefecture from Yunnan provinces of China. The origin of artificially cultivated Panax notoginseng are expanding from WenShan prefecture to other areas in Yunnan provinces. The dry roots and rhizomes of Panax notoginseng were position and has the functions of removing stasis, stopping blood and pain, activating blood 29,30 . 
Aucklandia lappa decne belonging to asteraceae perennial herb which mainly produced in Yunnan places like Lijiang Naxi Autonomous County and Ludian County, Zhaotong City. The origin of artificially cultivated Aucklandia lappa decne are distributed mainly over Sichuan,Yunnan, Guangxi and Guizhou provinces. Aucklandia lappa decne has the functions of stomach,tocolysis,relieving pain, regulating Qi and soothing the nerves. The application of OPPs in processes of rtificially cultivated Panax notoginseng and Aucklandia lappa decne were unavoidable, but there are few standard of detective pesticide residue in Panax notoginseng and Aucklandia lappa decne. For these reasons above, a rapid and simple analytical method with high accuracy and precision for determining 17 organophosphorus pesticide residues in Panax notoginseng and Aucklandia lappa decne by automated solid-phase extraction system and GC/MS was developed in this paper. The rapid and simple method for the simultaneous determination of 16 organophosphorus pesticide residues in Panax notoginseng and Aucklandia lappa decne was developed by comparing an ultrasonic extraction and homogenate extraction and optimizing purification conditions of automated solid-phase extraction system, which can meet the requirement of determining pesticide residues from Panax notoginseng and Aucklandia lappa decne.

\section{Experimental}

\subsection{Apparatus, Chemicals and Materials}

A Shimadzu GC/MS-QP2010 Ultra (Shimadzu,Japan), a J2 automated solid-phase extraction system (J2 Scientific,USA), a rotary evaporator (Switzerland, Germany), an electronic balance (Switzerland,Germany), a vortex mixer (Beijing, China),a universal pulverizer (Tianjin,China), an ultrasound cleaner (Shanghai,China) and the homogenate approach (Jiangsu,China) were used.

Acetone, dichloromethane,and n-hexane, all of analytical reagent grade, were from Tianjin University and were purchased from Tianjin Chemical Experiment Plant (Tianjin,China). Methylbenzene,acetonitrile,and methyl alcohol were all of HPLC grade and were purchased from Tedia (Fairfield,OH,USA). Anhydrous sodium sulfate and sodium chloride (Shanghai,China) were baked at $450^{\circ} \mathrm{C}$ for $4 \mathrm{~h}$ and stored in a desiccator before use. The standards of 17 OPPs (methamidophos, dichlorvos, acephate, omethoate, monocrotophos, phorate, phosphamidon, methyl parathion, fenitrothion, malathion, fenthion, chlorpyrifos, parathion, profenofos, triazophos, imidan, phosalone) with a solution concentration of 1000 mg/L were purchased from Quality Testing and Inspection Centre of the Ministry of Agriculture for Agricultural Products (Beijing, China).

Panax notoginseng and Aucklandia lappa decne samples were purchased from local markets (Yunnan, China). Solid-phase extraction cartridges (SPE cartridges) containing $\mathrm{C}_{18}, \mathrm{NH}_{4}$ and Florisil were purchased from Agela Technologies (Tianjin, China).

\section{$2.2 \mathrm{GC} / \mathrm{MS}$ conditions}

The separation was performed on an Rtx-5MS quartz capillary column ( $30 \mathrm{~m} \times 0.25 \mathrm{~mm}$, I.D. $0.25 \mu \mathrm{m})$ with a helium flow rate of $1.20 \mathrm{~mL}$.min ${ }^{-1}$ in a constant flow mode using the following oven temperature program: $70{ }^{\circ} \mathrm{C}(1 \mathrm{~min})$ to $250{ }^{\circ} \mathrm{C}(2 \mathrm{~min})$ at a rate of $8^{\circ} \mathrm{C} . \mathrm{min}^{-1}$ and $250{ }^{\circ} \mathrm{C}(2 \mathrm{~min})$ to 300 ${ }^{\circ} \mathrm{C}(10 \mathrm{~min})$ at a rate of $5^{\circ} \mathrm{C} \cdot \mathrm{min}^{-1}$. The injection inlet temperature was set at $250{ }^{\circ} \mathrm{C}$, and a $1.0 \mu \mathrm{L}$ volume was injected. Helium was the carrier gas. Using the splitless injection, the total analysis time was $45.5 \mathrm{~min}$. The mass spectrometer was operated in electron ionization mode with an ionizing energy of $70 \mathrm{eV}$, an ion source temperature of $230^{\circ} \mathrm{C}$, and an MS quad temperature of $280^{\circ} \mathrm{C}$. The scan ranged from $\mathrm{m} / \mathrm{z} 45$ to 500 ,and the solvent delay was $3.5 \mathrm{~min}$. Under the above GC-MS conditions,the total ion chromatogram (TIC) of 17 OPPs was obtained (Fig. 1).

\subsection{Standard solution preparation}

The $0.90 \mathrm{mg}$ (accurate to $0.01 \mathrm{mg}$ ) individual pesticide standards of 17 OPPs were weighed and redissolved in methylbenzene to make $10 \mathrm{~mL}$, which was to obtain the mixed standard reserve fluid of 17 OPPs. The standard stock mixtures were then diluted to $4.5 \mu \mathrm{g} \cdot \mathrm{mL}^{-1}$ with acetone.

The series of mixed standard working solutions for $2.00,1.00,0.50,0.25,0.10$, and $0.05 \mu \mathrm{g} . \mathrm{mL}^{-1}$ were made up by the dilution method step by step with acetone. The standard solutions were stored in the dark at $-4^{\circ} \mathrm{C}$ before use.

\subsection{Sample preparation}

The samples were pulverized into fine powders, sieved through 80 mesh and dried at $60^{\circ} \mathrm{C}$ for $4 \mathrm{~h}$. One gram of powder was then weighed accurately, and $60 \mathrm{~mL}$ of dichloromethane was added. The mixture was extracted for $2 \mathrm{~min}$ via homogenate extraction at $10000 \mathrm{r} / \mathrm{min}$, and then 1.0 $\mathrm{g}$ sodium sulfate anhydrous and $1.0 \mathrm{~g}$ sodium chloride were added. After filtration, the extracts were transferred into a flat-bottomed flask and condensed on a rotary evaporator at $40^{\circ} \mathrm{C}$ under vacuum until a volume of $5 \mathrm{~mL}$ was reached. The concentrated solutions were purified with Florisil solid-phase extraction cartridges by an automated solid-phase extraction system. The solid-phase extraction cartridges with the adsorbed extracts were eluted with $5 \mathrm{~mL}$ of methylbenzene-acetonitrile $(1: 3, \mathrm{v} / \mathrm{v})$ three times. The eluate solution was collected and evaporated until near dryness by a rotary evaporator with a water bath at $38^{\circ} \mathrm{C}$. Finally, the residues were resuspended in $1.00 \mathrm{~mL}$ of acetone and filtered through a $0.45 \mu \mathrm{m}$ PTFE filter for GC-MS analysis.

\subsection{Investigation of extraction and purification profiles}

\subsubsection{Investigation of ultrasonic extraction profiles}


The extraction technology implemented for the analysis of 17 OPPs from Panax notoginseng was under study, and the effects of factors such as extraction repetition and duration, extraction solvent and solvent volume on the extraction rate of 17 OPPs were examined through orthogonal experiments. The extraction repetition and duration, extraction solvent and solvent volume were screened, and an L9( $\left.3^{4}\right)($ Table 1$)$ orthogonal test was applied.

Table 1 The factors and levels of the ultrasonic extraction orthogonal experiment

\begin{tabular}{|llll|}
\hline $\begin{array}{l}\text { Factors } \\
\text { Levels }\end{array}$ & 1 & 2 & 3 \\
\hline A (Solvent) & Methanol & Acetonitrile & Dichloromethane \\
\hline B (Volume)/mL & 20 & 40 & 60 \\
\hline C (Repetitions) & 1 & 2 & 3 \\
\hline D (Duration)/min & 15 & 30 & 45 \\
\hline
\end{tabular}

\subsubsection{Investigation of homogenate extraction profiles}

The homogenate extraction approach of 17 OPPs from Panax notoginseng was under study, and the effects of factors such as time, revolution speed of homogenate extraction, extraction solvent and solvent volume on the extraction rate of 17 OPPs were examined through orthogonal experiments. The factors were screened, and an $\mathrm{L} 9\left(3^{4}\right)$ (Table 2$)$ orthogonal test was applied.

Table 2 The factor and level of the homogenate extraction orthogonal experiment

\begin{tabular}{|llll|}
\hline $\begin{array}{l}\text { Factors } \\
\text { Levels }\end{array}$ & 1 & 2 & 3 \\
\hline A (Solvent) & Methanol & Acetonitrile & Dichloromethane \\
\hline B (Volume)/ mL & 30 & 40 & 60 \\
\hline C (Revolution speed)/r/min & 10000 & 15000 & 20000 \\
\hline D (Time)/min & 2 & 3 & 5 \\
\hline
\end{tabular}

\subsubsection{Investigation of purification profiles}

The most effective purifying condition for 17 OPPs from Panax notoginseng was obtained experimentally by the orthogonal test design method. The influence of factors such as eluate, elution volume and times, and different solid-phase cartridges on the purification profiles were investigated. The factors were screened, and an $\mathrm{L} 9\left(3^{4}\right)$ (Table 3 ) orthogonal test was applied.

Table 3 The factors and levels of the purification orthogonal experiment

\begin{tabular}{|llll|}
\hline $\begin{array}{l}\text { Factors } \\
\text { Levels }\end{array}$ & 1 & 2 & 3 \\
\hline $\begin{array}{l}\text { A (Eluate) } \\
\text { (volume batching) }\end{array}$ & Acetone and N-hexane & Methylbenzene and Acetonitrile & Cyclohexane and Ethyl acetate \\
& $(1: 1, \mathrm{~V} / \mathrm{V})$ & $(1: 3, \mathrm{~V} / \mathrm{V})$ & $(1: 1, \mathrm{~V} / \mathrm{V})$ \\
\hline B (Volume)/mL & & & 20 \\
\hline C (Solid-phase cartridges) & $\mathrm{C}_{18}$ & 10 & Florisil \\
\hline D(times) & 1 & $\mathrm{NH}_{4}$ & 3 \\
\hline
\end{tabular}

\section{Results And Discussion}


Single standard solutions of 17 OPPs was monitored with the full-scan mode (SCAN) by GC/MS. One quantitative ion and two or three qualitative ions of each compound were confirmed.The detection method for 17 OPPs from $4.5 \mu \mathrm{g} / \mathrm{m}$ was established with the selected ion monitoring mode (SIM). The results are shown in Table 4.

Table 4 Parameters for the determination of OPPs in Panax notoginseng by GC/MS

\begin{tabular}{|c|c|c|c|}
\hline NO. & Pesticides & Retention time & $\begin{array}{l}\text { The qualitative and quantitative ions } \\
\text { (* the quantitative ions) }\end{array}$ \\
\hline & & $(\min )$ & $(\mathrm{m} / \mathrm{z})$ \\
\hline 1 & Methamidophos & 6.983 & $94^{\star}, 141,47,64$ \\
\hline 2 & Dichlorvos & 7.241 & $109 *, 79,185,145$ \\
\hline 3 & Acephate & 9.795 & $136 *, 94,47,125$ \\
\hline 4 & Omethoate & 11.817 & $156^{*}, 110,79,126$ \\
\hline 5 & Monocrotophos & 13.164 & $127 *, 97,67,192$ \\
\hline 6 & Phorate & 13.330 & $75^{\star}, 121,260,231$ \\
\hline 7 & Phosphamidon & 15.417 & $127 *, 264,15,205$ \\
\hline 8 & Methyl parathion & 16.965 & $109 *, 125,263,79$ \\
\hline 9 & Fenitrothion & 18.228 & $125^{\star}, 109,260,277$ \\
\hline 10 & Malathion & 18.712 & $173^{*}, 127,93,185$ \\
\hline 11 & Fenthion & 19.111 & $278^{*}, 125,169,245$ \\
\hline 12 & Chlorpyrifos & 19.146 & $314^{*}, 197,28,268$ \\
\hline 13 & Parathion & 19.259 & $291 *, 109,137,97$ \\
\hline 14 & Profenofos & 23.330 & $139 *, 208,337,269$ \\
\hline 15 & Triazophos & 26.695 & $161^{*}, 257,285,172$ \\
\hline 16 & Imidan & 30.185 & $160 *, 317,133,77$ \\
\hline 17 & Phosalone & 32.368 & $182 *, 121,154,367$ \\
\hline
\end{tabular}

\subsection{Selection of ultrasonic extraction and homogenate extraction conditions}

Based on the orthogonal test and multiple standard addition method, the data in Table 5 were obtained using the ultrasonic and homogenate extraction methods. Both of intuitionistic analysis and square-difference analysis is applied to analyze the data from Table 5, and the results are shown in Table 6, from which indicated that the dominant influence on the investigation factors for 17 OPPs from Panax notoginseng were the type of solvent used for the ultrasonic and homogenate extraction methods. For other factors with the ultrasonic and homogenate extraction, the effects on extraction efficiency were ranked as follows: $C>B>D$. The results showed the optimal conditions $\left(A_{3} B_{1} C_{1} D_{2}\right)$ for the extraction of 17 OPPs from Panax notoginseng by ultrasonic extraction were as follows: Panax notoginseng was extracted with approximately $20 \mathrm{~mL}$ of dichloromethane, and the extraction replication and duration times were one and 30 min, respectively. The results showed that the optimal conditions $\left(A_{3} B_{3} C_{1} D_{1}\right)$ for the extraction of 17 OPPs from Panax notoginseng by the homogenate extraction method were as follows: Panax notoginseng was extracted with approximately $60 \mathrm{~mL}$ of dichloromethane, and the extraction time was $2 \mathrm{~min}$ at $10000 \mathrm{r} / \mathrm{min}$.

Table 5 The recovery results of the extraction rates in ultrasonic and homogenate extraction by $L 9\left(3^{4}\right)(\%)$ 


\begin{tabular}{|c|c|c|c|c|c|c|c|c|c|}
\hline $\begin{array}{l}\text { Extraction } \\
\text { method }\end{array}$ & NO. & Methamidophos & Dichlorvos & Acephate & Omethoate & Monocrotophos & Phorate & Phosphamidon & $\begin{array}{l}\text { Methyl } \\
\text { parathion }\end{array}$ \\
\hline \multirow{9}{*}{$\begin{array}{l}\text { Ultrasonic } \\
\text { extraction }\end{array}$} & 1 & 67.95 & 77.32 & 75.59 & 68.27 & 64.52 & 73.27 & 69.66 & 93.43 \\
\hline & 2 & 67.89 & 76.7 & 75.45 & 67.88 & 63.98 & 72.9 & 69.39 & 91.36 \\
\hline & 3 & 59.72 & 74.43 & 75.14 & 67.42 & 61.66 & 70.51 & 67.99 & 58.99 \\
\hline & 4 & 62.95 & 62.9 & 65.65 & 64.26 & 78.27 & 79.55 & 71.33 & 75.83 \\
\hline & 5 & 67.96 & 63.71 & 66.84 & 65.50 & 81.51 & 76.62 & 72.3 & 76.83 \\
\hline & 6 & 61.37 & 61.31 & 70.96 & 66.18 & 70.89 & 65.36 & 63.99 & 86.66 \\
\hline & 7 & 67.68 & 76.72 & 75.56 & 67.63 & 63.32 & 72.32 & 69.23 & 95.43 \\
\hline & 8 & 64.31 & 62.85 & 72.56 & 63.98 & 81.52 & 65.29 & 69.54 & 85.13 \\
\hline & 9 & 65.13 & 61.58 & 73.82 & 65.86 & 80.44 & 62.48 & 69.56 & 86.84 \\
\hline \multirow{9}{*}{$\begin{array}{l}\text { Homogenate } \\
\text { extraction }\end{array}$} & 1 & 69.06 & 75.21 & 75.97 & 74.75 & 66.23 & 62.58 & 71.23 & 68.91 \\
\hline & 2 & 45.94 & 76.28 & 75.99 & 75.28 & 67.09 & 63.49 & 71.91 & 69.76 \\
\hline & 3 & 55.78 & 75.61 & 75.97 & 75.25 & 67.34 & 62.57 & 71.73 & 69.70 \\
\hline & 4 & 59.45 & 63.01 & 73.03 & 74.42 & 66.03 & 85.53 & 78.11 & 71.91 \\
\hline & 5 & 67.96 & 65.08 & 71.84 & 73.85 & 62.35 & 91.91 & 81.76 & 69.72 \\
\hline & 6 & 62.96 & 73.71 & 71.62 & 73.34 & 60.63 & 96.29 & 92.89 & 69.68 \\
\hline & 7 & 64.78 & 79.96 & 67.62 & 73.12 & 61.98 & 95.95 & 97.03 & 80.35 \\
\hline & 8 & 65.76 & 72.06 & 71.95 & 7346 & 63.33 & 97.32 & 80.52 & 72.88 \\
\hline & 9 & 66.99 & 62.22 & 74.38 & 73.93 & 65.03 & 80.88 & 67.52 & 65.04 \\
\hline
\end{tabular}

Table 5 The recovery results of the extraction rates in ultrasonic and homogenate extraction by $L 9\left(3^{4}\right)(\%)($ Continued) 


\begin{tabular}{|c|c|c|c|c|c|c|c|c|c|c|}
\hline $\begin{array}{l}\text { Extraction } \\
\text { method }\end{array}$ & NO. & Fenitrothion & Malathion & Fenthion & Chlorpyrifos & Parathion & Profenofos & Triazophos & Imidan & Phosalone \\
\hline \multirow{9}{*}{$\begin{array}{l}\text { Ultrasonic } \\
\text { extraction }\end{array}$} & 1 & 68.96 & 65.78 & 63.49 & 61.01 & 67.43 & 67.40 & 76.02 & 61.84 & 66.2 \\
\hline & 2 & 68.55 & 65.20 & 63.25 & 61.37 & 67.50 & 66.9 & 75.91 & 61.95 & 65.88 \\
\hline & 3 & 66.35 & 62.46 & 60.24 & 63.69 & 64.36 & 65.02 & 74.42 & 62.24 & 63.99 \\
\hline & 4 & 84.54 & 83.55 & 94.05 & 101.1 & 59.87 & 60.41 & 62.89 & 63.11 & 64.94 \\
\hline & 5 & 83.18 & 85.15 & 92.11 & 101.22 & 51.04 & 60.40 & 63.71 & 62.79 & 64.47 \\
\hline & 6 & 71.28 & 71.68 & 79.14 & 85.30 & 60.00 & 67.39 & 61.31 & 62.85 & 61.15 \\
\hline & 7 & 67.94 & 64.76 & 62.54 & 62.08 & 68.00 & 52.43 & 67.72 & 50.25 & 49.33 \\
\hline & 8 & 75.60 & 80.81 & 86.96 & 98.35 & 62.17 & 67.23 & 62.85 & 61.82 & 66.39 \\
\hline & 9 & 74.72 & 79.16 & 84.76 & 95.43 & 68.41 & 66.39 & 61.58 & 61.82 & 66.39 \\
\hline \multirow{9}{*}{$\begin{array}{l}\text { Homogenate } \\
\text { extraction }\end{array}$} & 1 & 27.00 & 67.2 & 64.34 & 60.04 & 63.66 & 68.33 & 48.78 & 61.87 & 66.38 \\
\hline & 2 & 43.98 & 67.94 & 65.06 & 61.63 & 62.54 & 67.59 & 60.87 & 61.91 & 66.41 \\
\hline & 3 & 45.66 & 67.44 & 64.12 & 61.08 & 63.14 & 67.19 & 67.38 & 61.85 & 66.42 \\
\hline & 4 & 74.25 & 91.53 & 90.27 & 100.74 & 117.98 & 46.72 & 67.37 & 34.77 & 60.38 \\
\hline & 5 & 66.94 & 94.66 & 101.5 & 107.36 & 131.25 & 68.35 & 56.89 & 59.19 & 45.67 \\
\hline & 6 & 64.85 & 100.25 & 103.16 & 110.81 & 135.37 & 68.38 & 67.37 & 57.94 & 65.76 \\
\hline & 7 & 70.70 & 88.31 & 85.18 & 101.53 & 128.93 & 68.27 & 67.37 & 49.57 & 48.77 \\
\hline & 8 & 70.23 & 93.24 & 97.05 & 103.67 & 125.92 & 68.37 & 67.35 & 49.76 & 54.32 \\
\hline & 9 & 81.83 & 79.52 & 97.98 & 89.73 & 105.79 & 68.41 & 67.36 & 61.82 & 61.54 \\
\hline
\end{tabular}

Table 6 The analysis results of intuitionistic analysis and square-difference analysis through orthogonal experiment with ultrasonic and homogenate extraction 


\begin{tabular}{|c|c|c|c|c|c|c|}
\hline Extraction method & NO. & $A$ & $\mathrm{~B}$ & $\mathrm{C}$ & D & Result \\
\hline \multirow[t]{13}{*}{ Ultrasonic extraction } & 1 & 1 & 1 & 1 & 1 & 30.5099 \\
\hline & 2 & 1 & 2 & 2 & 2 & 8.5829 \\
\hline & 3 & 1 & 3 & 3 & 3 & 6.4947 \\
\hline & 4 & 2 & 1 & 2 & 3 & 58.7942 \\
\hline & 5 & 2 & 2 & 3 & 1 & 24.9327 \\
\hline & 6 & 2 & 3 & 1 & 2 & 72.5786 \\
\hline & 7 & 3 & 1 & 3 & 2 & 53.5518 \\
\hline & 8 & 3 & 2 & 1 & 3 & 67.4066 \\
\hline & 9 & 3 & 3 & 2 & 1 & 57.8401 \\
\hline & Averaged 1 & 15.196 & 47.619 & 56.832 & 37.761 & - \\
\hline & Averaged 2 & 52.102 & 33.641 & 41.739 & 44.904 & - \\
\hline & Averaged 3 & 59.600 & 45.638 & 28.326 & 44.232 & - \\
\hline & Range & 44.404 & 13.978 & 28.506 & 7.143 & - \\
\hline \multirow[t]{14}{*}{ Homogenate extraction } & & $A$ & B & $\mathrm{C}$ & $\mathrm{D}$ & Result \\
\hline & 1 & 1 & 1 & 1 & 1 & 32.7974 \\
\hline & 2 & 1 & 2 & 2 & 2 & 13.8500 \\
\hline & 3 & 1 & 3 & 3 & 3 & 18.8223 \\
\hline & 4 & 2 & 1 & 2 & 3 & 4.0959 \\
\hline & 5 & 2 & 2 & 3 & 1 & 76.1613 \\
\hline & 6 & 2 & 3 & 1 & 2 & 102.4241 \\
\hline & 7 & 3 & 1 & 3 & 2 & 69.8418 \\
\hline & 8 & 3 & 2 & 1 & 3 & 78.4418 \\
\hline & 9 & 3 & 3 & 2 & 1 & 80.2859 \\
\hline & Averaged 1 & 21.823 & 35.578 & 71.221 & 63.082 & - \\
\hline & Averaged 2 & 60.894 & 56.151 & 32.744 & 62.039 & - \\
\hline & Averaged 3 & 76.190 & 67.177 & 54.942 & 33.787 & - \\
\hline & Range & 54.367 & 31.599 & 38.477 & 29.295 & - \\
\hline
\end{tabular}

\subsection{Selection of purification conditions}

Chinese medicinal materials contain a variety of matrices; thus, a clean-up procedure is necessary. ${ }^{[19]}$ Based on the orthogonal test and multiple standard addition method, the data in Table 7 are the purification profiles of the orthogonal $\mathrm{L} 9\left(3^{4}\right)$ method implemented. The recovery results of the purification profiles are shown in Table 7. The results of the intuitionistic analysis and square-difference analysis are shown in Table 8 . The results of the experiments and the intuitionistic analysis and square-difference analysis of experimental (Table 7 and Table 8 ) results showed that the dominant influence on the purification recoveries of 17 OPPs was the type of eluting solvent. For other factors, the effects of the purification profiles were ranked as follows: $B>D>C$. The optimal purification conditions were a combination of $A_{3} B_{3} C_{1} D_{1}$ that 3 extractions with approximately 5 mL of methylbenzene-acetonitrile (1:3, V/V) each time from Florisil solid-phase cartridges met the requirement for the purification of the 17 OPPs.

Table 7 The recovery results of the purification profiles by $L 9\left(3^{4}\right)(\%)$ 


\begin{tabular}{|llllllll|}
\hline NO. & Methamidophos & Dichlorvos & Acephate & Omethoate & Monocrotophos & Phorate & Phosphamidon \\
\hline 1 & 55.99 & 65.76 & 62.46 & 64.34 & 54.51 & 53.54 & 57.99 \\
\hline 2 & 51.77 & 63.56 & 73.44 & 61.17 & 51.79 & 50.77 & 54.93 \\
\hline 3 & 62.49 & 65.95 & 63.49 & 73.91 & 52.06 & 54.38 & 54.24 \\
\hline 4 & 56.39 & 66.31 & 58.79 & 60.14 & 150.58 & 53.71 & 88.13 \\
\hline 5 & 50.91 & 66.48 & 63.25 & 53.36 & 88.13 & 54.52 & 96.50 \\
\hline 6 & 55.11 & 51.99 & 55.65 & 63.04 & 65.29 & 53.79 & 69.44 \\
\hline 7 & 51.18 & 63.02 & 50.80 & 54.65 & 63.48 & 54.36 & 74.60 \\
\hline 8 & 56.66 & 67.46 & 65.34 & 64.26 & 55.13 & 54.78 & 73.59 \\
\hline 9 & 57.62 & 67.07 & 65.06 & 62.75 & 54.55 & 54.67 & 52.88 \\
\hline
\end{tabular}

Table 7 The recovery results of the purification profiles by L9( $\left.3^{4}\right)(\%)$ (Continued)

\begin{tabular}{|lllllllllll|}
\hline No. & $\begin{array}{l}\text { Methyl } \\
\text { parathion }\end{array}$ & Fenitrothion & Malathion & Fenthion & Chlorpyrifos & Parathion & Profenofos & Triazophos & Imidan & Phosalone \\
\hline 1 & 58.47 & 59.34 & 57.59 & 54.43 & 51.41 & 52.05 & 53.84 & 55.65 & 52.78 & 54.15 \\
\hline 2 & 56.54 & 51.29 & 53.49 & 50.25 & 51.02 & 54.46 & 50.52 & 50.62 & 54.57 & 50.31 \\
\hline 3 & 59.41 & 75.46 & 58.56 & 55.44 & 52.83 & 51.31 & 56.61 & 55.09 & 52.17 & 55.47 \\
\hline 4 & 54.17 & 79.81 & 54.86 & 55.74 & 108.09 & 51.69 & 50.89 & 58.53 & 52.34 & 53.79 \\
\hline 5 & 55.42 & 71.84 & 52.56 & 55.80 & 51.77 & 51.61 & 54.71 & 55.72 & 52.33 & 54.82 \\
\hline 6 & 51.95 & 70.51 & 53.82 & 55.91 & 136.44 & 52.31 & 56.58 & 53.31 & 52.25 & 54.01 \\
\hline 7 & 76.11 & 97.41 & 56.22 & 54.64 & 60.13 & 50.87 & 64.04 & 60.66 & 55.63 & 52.50 \\
\hline 8 & 57.47 & 62.31 & 57.61 & 55.97 & 53.58 & 51.20 & 55.74 & 56.39 & 52.32 & 54.78 \\
\hline 9 & 57.78 & 81.35 & 58.05 & 55.94 & 53.67 & 50.96 & 57.56 & 56.57 & 51.97 & 55.66 \\
\hline
\end{tabular}

Table 8 The analysis results of intuitionistic analysis and square-difference analysis through orthogonal experiment with automated solidphase extraction system

\begin{tabular}{|llllll|}
\hline NO. & A & B & C & D & Result \\
\hline 1 & 1 & 1 & 1 & 1 & 104.7890 \\
\hline 2 & 1 & 2 & 2 & 2 & 104.7031 \\
\hline 3 & 1 & 3 & 3 & 3 & 106.3391 \\
\hline 4 & 2 & 1 & 2 & 3 & 114.3657 \\
\hline 5 & 2 & 2 & 3 & 1 & 108.2106 \\
\hline 6 & 2 & 3 & 1 & 2 & 108.4004 \\
\hline 7 & 3 & 1 & 3 & 2 & 111.6991 \\
\hline 8 & 3 & 2 & 1 & 3 & 106.3132 \\
\hline 9 & 3 & 3 & 2 & 1 & 105.2920 \\
\hline Averaged 1 & 105.277 & 110.285 & 106.501 & 106.097 & - \\
\hline Averaged 2 & 110.326 & 106.409 & 108.120 & 108.268 & - \\
\hline Averaged 3 & 107.767 & 106.676 & 108.750 & 109.006 & - \\
\hline Range & 5.049 & 3.876 & 2.249 & 2.909 & - \\
\hline
\end{tabular}




\subsubsection{Calibration curves, determination coefficients and LODs}

To confirm that the optimized method was suitable for application, precision, recovery, linear range and detection limits (LODs) were evaluated for the analytical approach developed using samples of Panax notoginseng. Quantitative analysis was achieved using an external standard. The calibration curve was obtained by analyzing blank Panax notoginseng samples spiked with pesticides at the same levels (Table 9). For detection of the 17 OPPs by GC/MS, the LODs were $0.12 \sim 0.52 \times 10^{-3} \mu \mathrm{g}$. The acceptable linearity was indicated by the determination coefficients, which ranged from 0.9928 to 0.9999 .

Table 9 Calibration curves, determination coefficients and LODs of the pesticides analyzed by GC/MS

\begin{tabular}{|c|c|c|c|c|c|}
\hline NO. & Pesticide & Calibration curve & Determination coefficient/r & $\begin{array}{l}\text { Linear range } \\
(\mathrm{g} / \mathrm{mL})\end{array}$ & $\begin{array}{l}\text { LODs } \\
\left(\times 10^{-3} \mu \mathrm{g}\right)\end{array}$ \\
\hline 1 & Methamidophos & $Y=1578831 X+125793.7$ & 0.9941 & $0.005 \rrbracket 2.0$ & 0.12 \\
\hline 2 & Dichlorvos & $Y=2852786 X+455151.9$ & 0.9945 & $0.005 \rrbracket 2.0$ & 0.17 \\
\hline 3 & Acephate & $Y=662784.3 X+104183.2$ & 0.9986 & $0.005 \rrbracket 2.0$ & 0.2 \\
\hline 4 & Omethoate & $Y=585230.1 X+82190.18$ & 0.9928 & $0.005 \rrbracket 2.0$ & 0.31 \\
\hline 5 & Monocrotophos & $Y=1810437 X+138459.1$ & 0.9947 & $0.005 \rrbracket 2.0$ & 0.26 \\
\hline 6 & Phorate & $Y=3379836 X+149327.7$ & 0.9980 & $0.005 \rrbracket 2.0$ & 0.38 \\
\hline 7 & Phosphamidon & $Y=296661.7 X+36655.06$ & 0.9909 & $0.005 \rrbracket 2.0$ & 0.21 \\
\hline 8 & Methyl parathion & $Y=1037505 X+100506.8$ & 0.9973 & $0.005 \rrbracket 2.0$ & 0.46 \\
\hline 9 & Fenitrothion & $Y=906542.2 X+315367.7$ & 0.9954 & $0.005 \rrbracket 2.0$ & 0.52 \\
\hline 10 & Malathion & $Y=1514504 X+128704.2$ & 0.9967 & $0.005 \bowtie 2.0$ & 0.32 \\
\hline 11 & Fenthion & $Y=2456014 X+139159.0$ & 0.9973 & $0.005 \rrbracket 2.0$ & 0.45 \\
\hline 12 & Chlorpyrifos & $Y=661122.3 X+28795.83$ & 0.9971 & $0.005 \bowtie 2.0$ & 0.13 \\
\hline 13 & Parathion & $Y=661122.3 X+28795.83$ & 0.9971 & $0.005 \bowtie 2.0$ & 0.37 \\
\hline 14 & Profenofos & $Y=635449.9 X-4061.267$ & 0.9999 & $0.005 \bowtie 2.0$ & 0.42 \\
\hline 15 & Triazophos & $Y=1102994 X+73458.66$ & 0.9974 & $0.005 \llbracket 2.0$ & 0.18 \\
\hline 16 & Imidan & $Y=6699876 X-109404.20$ & 0.9987 & $0.005 \bowtie 2.0$ & 0.41 \\
\hline 17 & Phosalone & $Y=1403894 X+81005.27$ & 0.9969 & $0.005 \bowtie 2.0$ & 0.22 \\
\hline
\end{tabular}

\subsubsection{Results of methodology validation}

Under the optimized extraction, purification and detection conditions for 17 OPPs in Panax notoginseng determined by orthogonal design, two analytical methods were developed. In the first method, Panax notoginseng was extracted by ultrasonic extraction. The extracts were cleaned up by an automated solid-phase extraction system and determined by GC/MS. The second method used homogenate extraction. Experiments in which standards were added to the samples to determine the recovery rate were performed. The standard recovery test results for the two methods and calculation of the recovery rate are shown in Table 10 . The average recoveries of the first method ranged from $43.37 \%$ to $85.63 \%$, with the relative standard deviations (RSDs) of $0.07 \sim 0.17 \%(n=3)$. The average recoveries of the second method ranged from $67.88 \%$ to $96.70 \%$, with $\mathrm{RSDs}$ of $0.05 \sim 0.14 \%(n=3)$. The results showed that the average recoveries of the first method were often relatively lower the second method. Therefore, the analytical method was developed by homogenate extraction and automated solid-phase extraction system(the specific condition shown in 3.2 and 3.3). The average recovery (67.88\%) of methyl parathion was just below $70 \%$. The average recoveries of other OPPs ranged from $70 \%$ to $120 \%$, with RSD of less than $10 \%$. So the established analytical method can be applied to determining 16 organophosphorus pesticide residues in Panax notoginseng. Similarly,to further verify the viability of the method for Aucklandia lappa decne,recovery experiments were carried out,the results were satisfactory. The rapid and simple method for the simultaneous determination of 16 organophosphorus pesticide residues in Panax notoginseng and Aucklandia lappa decne was developed,which can meet the requirement of determining pesticide residues from Panax notoginseng and Aucklandia lappa decne. 
Table 10 The average recoveries of two analytical methods

Page $11 / 15$ 


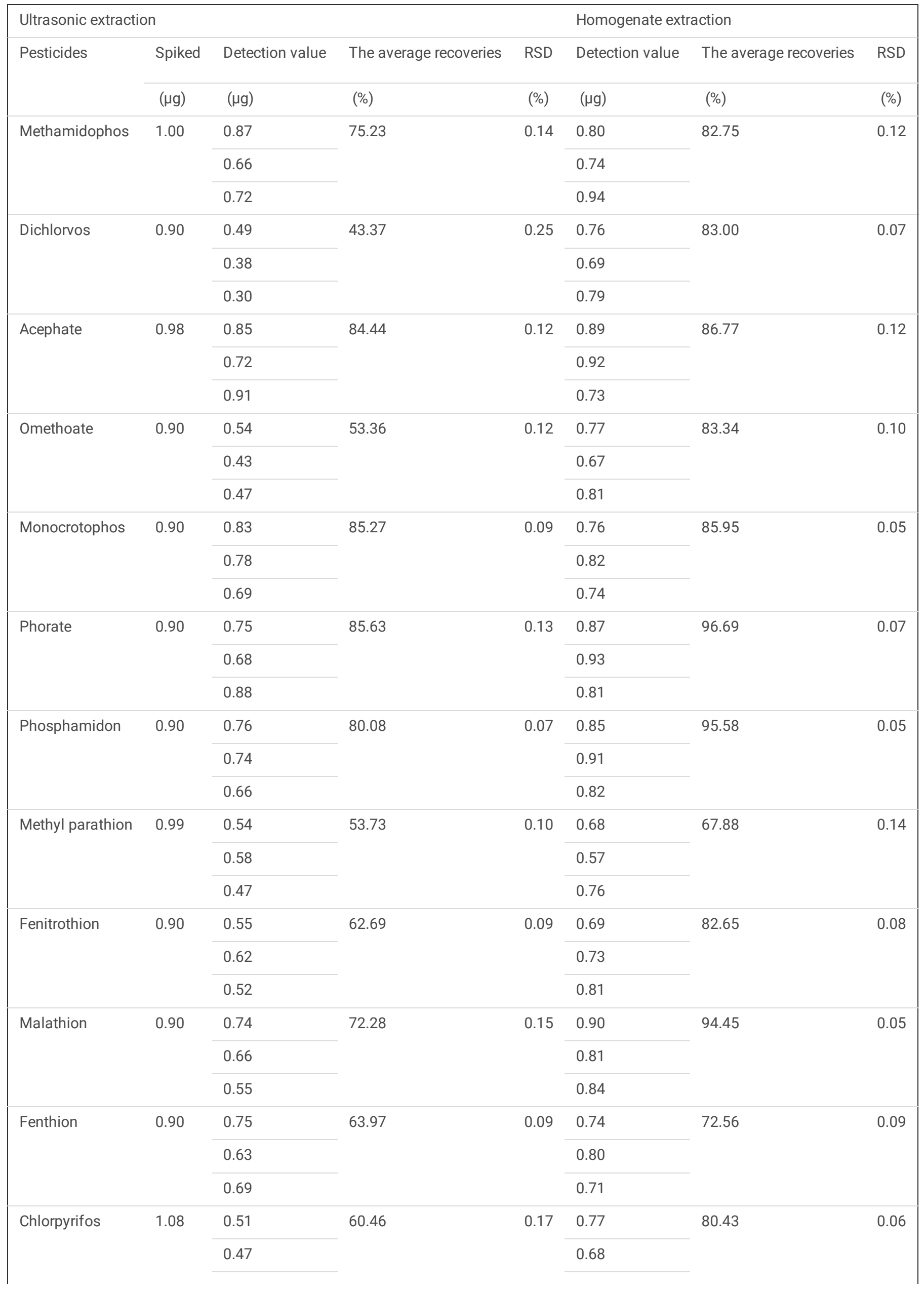

Page 12/15 


\begin{tabular}{|c|c|c|c|c|c|c|c|}
\hline & & 0.65 & & & 0.72 & & \\
\hline \multirow[t]{3}{*}{ Parathion } & \multirow[t]{3}{*}{0.90} & 0.84 & \multirow[t]{3}{*}{84.44} & \multirow[t]{3}{*}{0.09} & 0.76 & \multirow[t]{3}{*}{78.16} & \multirow[t]{3}{*}{0.08} \\
\hline & & 0.73 & & & 0.70 & & \\
\hline & & 0.71 & & & 0.65 & & \\
\hline \multirow[t]{3}{*}{ Profenofos } & \multirow[t]{3}{*}{0.90} & 0.58 & \multirow[t]{3}{*}{59.67} & \multirow[t]{3}{*}{0.23} & 0.85 & \multirow[t]{3}{*}{86.35} & \multirow[t]{3}{*}{0.09} \\
\hline & & 0.40 & & & 0.77 & & \\
\hline & & 0.63 & & & 0.71 & & \\
\hline \multirow[t]{3}{*}{ Triazophos } & \multirow[t]{3}{*}{0.90} & 0.71 & \multirow[t]{3}{*}{67.08} & \multirow[t]{3}{*}{0.15} & 0.93 & \multirow[t]{3}{*}{96.70} & \multirow[t]{3}{*}{0.06} \\
\hline & & 0.56 & & & 0.82 & & \\
\hline & & 0.54 & & & 0.86 & & \\
\hline \multirow[t]{3}{*}{ Imidan } & \multirow[t]{3}{*}{0.90} & 0.62 & \multirow[t]{3}{*}{74.95} & \multirow[t]{3}{*}{0.07} & 0.76 & \multirow[t]{3}{*}{90.38} & \multirow[t]{3}{*}{0.06} \\
\hline & & 0.71 & & & 0.83 & & \\
\hline & & 0.69 & & & 0.85 & & \\
\hline \multirow[t]{3}{*}{ Phosalone } & \multirow[t]{3}{*}{0.90} & 0.73 & \multirow[t]{3}{*}{82.65} & \multirow[t]{3}{*}{0.10} & 0.79 & \multirow[t]{3}{*}{88.20} & \multirow[t]{3}{*}{0.07} \\
\hline & & 0.68 & & & 0.74 & & \\
\hline & & 0.82 & & & 0.85 & & \\
\hline
\end{tabular}

\subsection{The sample test}

The established analytical method can be applied to determining 16 organophosphorus pesticide residues in Panax notoginseng and Aucklandia lappa decne from yunnan.The test indicated that Methamidophos, Dichlorvos, Acephate, Omethoate, Monocrotophos,Phorate,Phosphamidon,Fenitrothion,Malathion,Fenthion,Chlorpyrifos,Parathion,Profenofos, Triazophos,Imidan and Phosalone in Panax notoginseng and Aucklandia lappa decne were not detected.

\section{Conclusion}

Two sample pretreatment methods for the extraction of 16 OPPs from the traditional Chinese medicinal herb Panax notoginseng and Aucklandia lappa decne were developed. A comparison of the two methods of analyzing 16 OPPs in Panax notoginseng was performed. We can see that the homogenate extraction method is effective, with a high extraction rate and shortened extraction time. Therefore, a fast and sensitive multi-residue analysis method was developed for the determination of organophosphorus pesticide residues in Panax notoginseng and Aucklandia lappa decne by a homogenate extraction approach coupled with automated solid-phase extraction system and GC/MS. Under the optimum conditions, the results of the homogenate approach were compared with the ultrasonic extraction method, and the homogenate approach was used to extract the samples. The average recoveries of the method ranged from $72.56 \%$ to $96.70 \%$, and RSDs were $0.05 \sim 0.14 \%$, which meets the requirements for the determination of 16 organophosphorus pesticide residues in Panax notoginseng and Aucklandia lappa decne.

\section{Declarations}

\section{Acknowledgments}

This work was financially supported by the Department of Science and Technology of Yunnan Province (Grant No. $2012 \mathrm{FD} 053$ and No. 2013FZ121) and the National Natural Science Foundation of China (Grant No. 61361002 and Grant No. 21366011).

\section{Conflicts of interest}

There are no conflicts of interest to declare.

\section{References}

1. J.P.S. Gill, J.S. Bedi, R. Singh, M.N. Fairoze, R.A. Hazarika, A. Gaurav, S.K. Satpathy, A.S. Chauhan, J. Lindahl, D. Grace, A. Kumar, M. Kakkar. Pesticide Residues in Peri-Urban Bovine Milk from India and Risk Assessment: A Multicenter Study. Sci. Rep. 10, 8054 (2020). doi:10.1038/s41598-020-65030-z. 
2. H. Musarurwa, N.T. Tavengwa. Emerging green solvents and their applications during pesticide analysis in food and environmental samples. Talanta 223, 121507(2021). doi:10.1016/j.talanta.2020.121507.

3. X. Yuan, D. Zhang, X. Zhu, H. Liu, B. Sun. Triple-dimensional spectroscopy combined with chemometrics for the discrimination of pesticide residues based on ionic liquid-stabilized Mn-ZnS quantum dots and covalent organic frameworks. Food Chem. 128299 (2020). doi:10.1016/j.foodchem.2020.128299.

4. L. Kuang, Y. Hou, F. Huang, H. Hong, H. Sun, W. Deng, H. Lin. Pesticide residues in breast milk and the associated risk assessment: A review focused on China. Sci Total Environ 727, 138412(2020). doi:10.1016/j.scitotenv.2020.138412.

5. W.C. Griffith, E.M. Vigoren, M.N. Smith, T. Workman, B. Thompson, G.D. Coronado, E.M. Faustman. Application of improved approach to evaluate a community intervention to reduce exposure of young children living in farmworker households to organophosphate pesticides. $J$. Expo. Sci. Environ. Epidemiol. 29, 358-365(2019). doi:10.1038/s41370-018-0028-y.

6. L. Kuang, Y. Hou, F. Huang, A. Guo, W. Deng, H. Sun, L. Shen, H. Lin, H. Hong. Pesticides in human milk collected from Jinhua, China: Levels, influencing factors and health risk assessment. Ecotoxicol Environ Saf 205, 111331(2020). doi:10.1016/j.ecoenv.2020.111331.

7. P.M. Frugeri, M.H. da Silva Cavalcanti, A.C. do Lago, E.C. Figueiredo, C.R.T. Tarley, C. Wisniewski, P.O. Luccas,. Magnetic restricted-access carbon nanotubes for the extraction/pre-concentration of organophosphates from food samples followed by spectrophotometric determination. Spectrochim. Acta, Part A 241, 118632 (2020). doi:10.1016/j.saa.2020.118632.

8. H. Liu, X. Bai, X. Pang. Intercity variability and local factors influencing the level of pesticide residues in marketed fruits and vegetables of China. Sci Total Environ 700, 134481 (2020). doi:10.1016/j.scitotenv.2019.134481.

9. Z. Li, Y. Zhang, Q. Zhao, C. Wang, Y. Cui, J. Li, A. Chen, G. Liang, B. Jiao. Occurrence, temporal variation, quality and safety assessment of pesticide residues on citrus fruits in China. Chemosphere 258, 127381(2020). doi:10.1016/j.chemosphere.2020.127381.

10. J. He, H. He, Z. Yan, F. Gao, X. Zheng, J. Fan, Y. Wang. Comparative analysis of freshwater species sensitivity distributions and ecotoxicity for priority pesticides: Implications for water quality criteria. Ecotoxicol Environ Saf 176, 119-124(2019). doi:10.1016/j.ecoenv.2019.03.087.

11. A. Heshmati, A. Nili-Ahmadabadi, A. Rahimi, A. Vahidinia, M. Taheri. Dissipation behavior and risk assessment of fungicide and insecticide residues in grape under open-field, storage and washing conditions. J. Cleaner Prod. 270, 122287 (2020). doi:10.1016/j.jclepro.2020.122287.

12. S. Omar, I.A. Bahemia, L. Toerien, K.M. San Pedro, A.B. Khan. A retrospective comparison of the burden of organophosphate poisoning to an Intensive Care Unit in Soweto over two separate periods. Afr. J. Emerg Med (2020). doi:10.1016/j.afjem.2020.09.007.

13. T. Liu, S. Xu, S. Lu, P. Qin, B. Bi, H. Ding, Y. Liu, X. Guo, X. Liu. A review on removal of organophosphorus pesticides in constructed wetland: Performance, mechanism and influencing factors. Sci. Total Environ. 651, 2247-2268(2019). doi:10.1016/j.scitotenv.2018.10.087.

14. R. Pandiselvam, R. Kaavya, Y. Jayanath, K. Veenuttranon, P. Lueprasitsakul, V. Divya, A. Kothakota, S.V. Ramesh. Ozone as a novel emerging technology for the dissipation of pesticide residues in foods-a review. Trends Food Sci. Technol. 97, 38-54(2020). doi:10.1016/j.tifs.2019.12.017.

15. W.G. Rong, H. Guo, Y. Hong. Current research status in China on pesticide contamination of plant material used in making Chinese herbal medicines. Agrochem icals. 45, 302-308 (2006). doi:10.16820/j.cnki.1006-0413.2006.05.004.

16. Pharmacopoeia of the People's Republic of China.(2005 ed.). Beijing: Chemical Industry Press, 2005.

17. L.L. Hao, J. Xue,. Multiresidue analysis of organochlorine pesticides and the application to Chinese herbal medicine. China J. Chin. Mater. Med. 30, 405-409 (2005). doi:10.3321/j.issn:1001-5302.2005.06.001.

18. A. Lawal, R.C.S. Wong, G.H. Tan, L.B. Abdulra'uf, A.M.A. Alsharif. Multi-pesticide Residues Determination in Samples of Fruits and Vegetables Using Chemometrics Approach to QuEChERS-dSPE Coupled with lonic Liquid-Based DLLME and LC-MS/MS. Chromatographia 81, 759-768 (2018). doi:10.1007/s10337-018-3511-7.

19. H. Musarurwa, L. Chimuka, V.E. Pakade, N.T. Tavengwa. Recent developments and applications of QuEChERS based techniques on food samples during pesticide analysis. J. Food Compos. Anal. 84, 103314(2019). doi:10.1016/j.jfca.2019.103314.

20. Z. Chen, Q. Li, T. Yang, Y. Zhang, M. He, H. Zeng, X. Mai, Y. Liu, H. Fan. Sequential extraction and enrichment of pesticide residues in Longan fruit by ultrasonic-assisted aqueous two-phase extraction linked to vortex-assisted dispersive liquid-liquid microextraction prior to high performance liquid chromatography analysis. J. Chromatogr. A 1619, 460929(2020). doi:10.1016/j.chroma.2020.460929.

21. L. Drabova, G. Alvarez-Rivera, M. Suchanova, D. Schusterova, J. Pulkrabova, M. Tomaniova, V. Kocourek, O. Chevallier, C. Elliott, J. Hajslova. Food fraud in oregano: Pesticide residues as adulteration markers. Food Chem. 276, 726-734(2019). doi:10.1016/j.foodchem.2018.09.143.

22. O. Golge, B. Kabak. Pesticide Residues in Table Grapes and Exposure Assessment. J. Agric. Food. Chem. 66, 1701-1713 (2018). doi:10.1021/acs.jafc.7b05707.

23. B. Maestroni, N. Besil, A. Bojorge, N. Gérez, A. Pérez-Parada, A. Cannavan, H. Heinzen, M.V. Cesio. Optimization and validation of a single method for the determination of pesticide residues in Peumus boldus Molina leaves using GC-MSD, GC-MS/MS and LC-MS/MS. J. Appl. Res. Med. Aromat. Plants 18, 100254 (2020). doi:10.1016/j.jarmap.2020.100254.

24. R.X. Li, M.M. Li, T. Wang, T.L. Wang, J.Y. Chen, F. Francis, B. Fan, Z.Q. Kong, X.F. Dai. Screening of pesticide residues in Traditional Chinese Medicines using modified QuEChERS sample preparation procedure and LC-MS/MS analysis. J. Chromatogr. B Analyt. Technol. Biomed. Life

Page $14 / 15$ 
Sci. 1152, 122224 (2020). doi:10.1016/j.jchromb.2020.122224.

25. X. Chen, Z. Bian, H. Hou, F. Yang, S. Liu, G. Tang, Q. Hu. Development and validation of a method for the determination of 159 pesticide residues in tobacco by gas chromatography-tandem mass spectrometry. J. Agric. Food. Chem. 61, 5746-5757(2013). doi:10.1021/jf400887x.

26. J. Wang, H.L. Duan, L. Fan, J. Zhang, Z.Q. Zhang. A magnetic fluorinated multi-walled carbon nanotubes-based QuEChERS method for organophosphorus pesticide residues analysis in Lycium ruthenicum Murr. Food Chem. 338, 127805(2021). doi:10.1016/j.foodchem.2020.127805.

27. M. Yang, L. Wang. Advances in Techniques on Analysis and Removal of Pesticide Residues in Traditional Chinese Herbal Medicines. World Sci. Technol.10, 107-112 (2008). doi:10.1016/s1876-3553(09)60005-3.

28. S.C. Yan, J. Ma, Q.Q. Huang, A. Mani, Y.L. Cai, C.X. Chen, C.P. Yin, Y.C. Ma. Simultaneous Quantification of a Herbal Combination of Pueraria lobata, Salvia miltiorrhiza and Panax notoginseng by Rapid Resolution Liquid Chromatography. Nat. Prod. Commun. 12, 1501-1506 (2017). doi:10.1177/1934578x1701200930.

29. N. Wu, Y.D. Zhang, W. Liu, P. Yi, Z.F. Wang, D.S. Huang. The Determination of Eight Pesticides in Panax notoginseng of Chinese Traditional Medicine Using Solid-Phase Extraction and Gas Chromatography-Mass Spectrometry. Adv. Mater. Res.485, 68-71 (2012). doi:10.4028/www.scientific.net/AMR.485.68.

30. N. Wu, B. Zhou, H.P. Yan, S.J. Xu, Y.H. Long, W. Liu. Determination of Hexachlorocyclohexane (HCH) in Panax notoginseng of Chinese Traditional Medicine by Gas Chromatography/Mass Spectrometry. Advanced Materials Research 830, $422-425$ (2013). doi:10.4028/www.scientific.net/AMR.830.422.

\section{Figures}

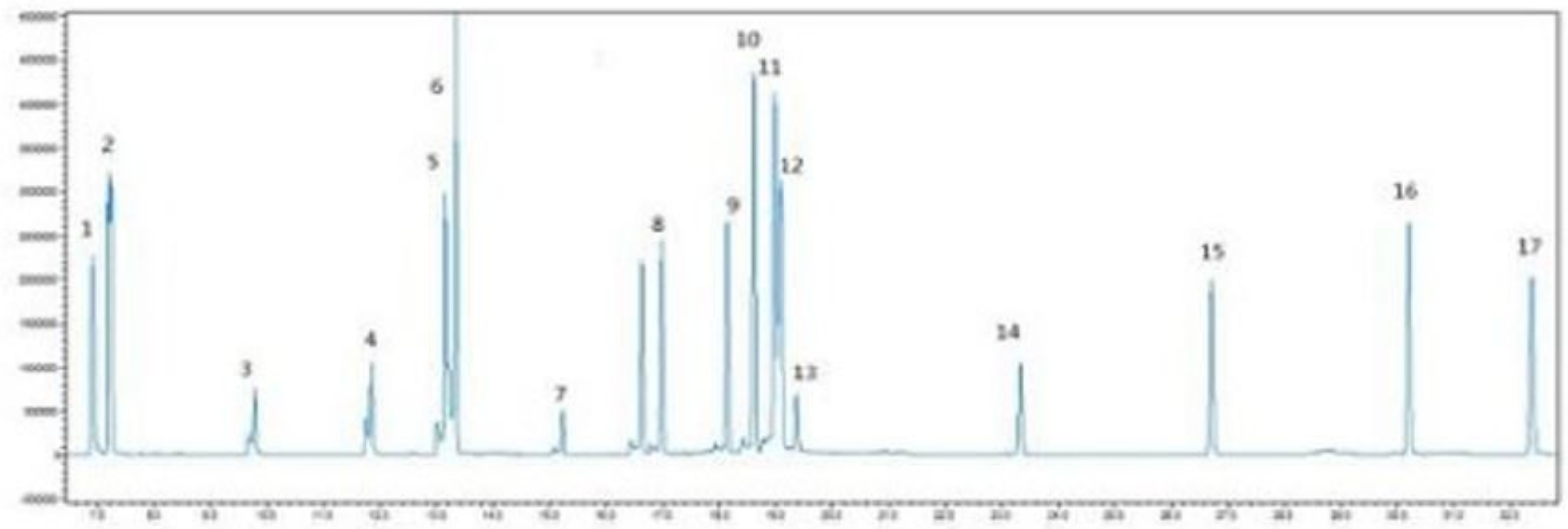

Figure 1

The TIC of 17 OPPs 